NEURODEGENERATION

\section{CREB, the cell-death solution}

The cyclic-AMP-responsive element (CRE)-binding protein (CREB) family of transcription factors has been implicated in many processes, including memory formation and maintenance, circadian rhythms and cell survival in vitro. Two papers now show a crucial role for CREB-family members in cell survival in vivo in both the central (CNS) and peripheral nervous systems, and provide further support for the postulated role of CREB in the pathogenesis of polyglutamine diseases.

There are three members of the CREB family - CREB, CRE-modulatory protein (CREM) and activating transcription factor 1 (ATF1). These proteins activate transcription by binding to CREs in the promoter regions of target genes. Mantamadiotis et al. assessed the role of CREB in neuronal survival by knocking out Creb1 specifically in the developing brain or in the postnatal forebrain of mice. Although they found no obvious phenotype in these animals, they did observe an upregulation of CREM (but not of ATF1). To investigate whether this upregulation compensated for the loss of CREB, they looked at the effect of knocking out $\mathrm{Creb} 1$ in a $\mathrm{Crem}^{--}$background, and found extensive apoptosis of postmitotic neurons during development, indicating that the two transcription factors might have a redundant effect on cell survival.

Intriguingly, Mantamadiotis et al. also found specific and progressive postnatal neurodegeneration in the CA1 and dentate gyrus of the hippocampus, and in the dorsal striatum. It is possible that the similarity between the degeneration pattern in the dorsal striatum that was observed in these mice and that seen in Huntington's disease is more than a coincidence, as there is some evidence that polyglutamine repeats can sequester some key CREB effectors.

It would have been convenient to draw simple conclusions about the redundant function of CREB and
CREM in neuronal survival, and to move on to think about their possible downstream genes. But in a related paper, Lonze et al. remind us of the complexity of CREB regulation. They show that the redundant function that was observed in the CNS is not mirrored in the periphery, by reporting that mice carrying a null mutation in Creb1 show excessive apoptosis and degeneration of sensory neurons during development. In addition, $\mathrm{Creb}^{-1-}$ cultured sympathetic and sensory neurons showed impaired neurotrophindependent survival and axonal extension. Like Mantamadiotis et al., Lonze and co-workers did not find abnormal degeneration in the CNS during development; however, they were not able to study mice after birth, because the $\mathrm{Creb}^{-/-}$animals die perinatally. Nevertheless, Lonze et al. have succeeded in providing us with clues about the possible mechanism of action of this transcription factor. They showed that CREB is phosphorylated on its main regulatory residue - serine 133 - during a period in which neurons from the superior cervical and dorsal root ganglia are dependent on nerve growth factor and neurotrophin 3 for survival.

It remains possible that ATF1 could compensate for the lack of CREB and CREM in the CNS, and that both CREM and ATF1 partially compensate for CREB in the periphery. However, the results clearly show that CREB-family members are crucial for neuronal survival, and these data add fuel to the idea that the characteristic neurodegeneration in Huntington's disease might be related to disruption of the CREB-dependent expression of cell-survival factors.

Michael Stebbins Assistant Editor, Nature Genetics

\section{(1) References and links} ORIGINAL RESEARCH PAPERS Mantamadiotis, T. et al. Disruption of CREB function in brain leads to neurodegeneration. Nature Genet. 31, 47-54 (2002) | Lonze, B. E. et al. Apoptosis, axonal growth defects, and degeneration of peripheral neurons in mice lacking CREB. Neuron $\mathbf{3 4}$, 371-385 (2002)

\section{ION CHANNELS}

\section{The Sigma K Fraternity}

The so-called sigma receptor has been something of a mystery molecule for some time. First described as an opioid receptor, it was later found to interact with many types of drug and to affect the nervous, endocrine and immune systems. One example of sigma receptor function is its ability to mediate the modulatory effects of psychotropic compounds on some ion channels. New data from Aydar et al. indicate that this

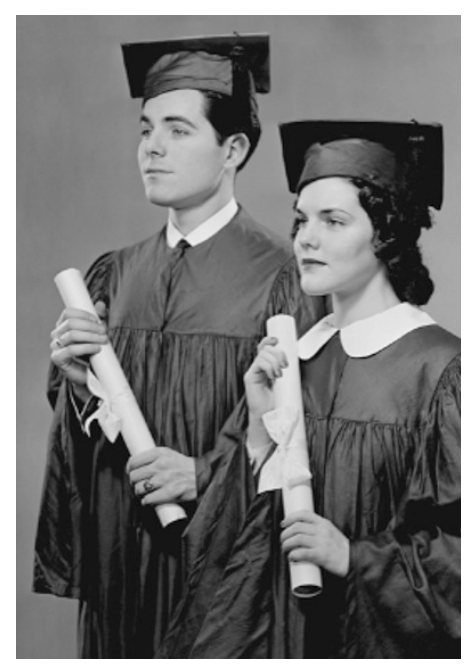
modulation might depend on direct protein-protein interactions with the channel.

The authors expressed the voltage-gated $\mathrm{K}^{+}$channels Kv1.4 and Kv1.5 in Xenopus oocytes, and found that $\mathrm{K}^{+}$currents were affected by co-expression of the sigma receptor. The nature of the modulation depended on whether a sigma receptor ligand was present or not: in the absence of ligand, the receptor accelerated voltage-dependent channel inactivation and reduced current amplitude; in its presence, the receptor decreased the peak current even further.

Co-immunoprecipitation studies in pituitary cells (in which sigma receptors and $\mathrm{K}^{+}$channels are known to interact) allowed Aydar et al. to conclude that the two proteins were part of the same molecular complex. It is unclear whether their interaction is direct; however, as the modulatory effect was preserved in the oocytes, a direct contact is likely, unless any further interacting proteins are also naturally present in their expression system. Which parts of the sigma receptor are involved in this interaction? We don't yet know, but the authors made two observations that might help us to answer this question. First, the receptor has two transmembrane domains (instead of one, as was previously thought). Second, both the amino and carboxyl termini of the protein face the cytoplasm. Gaining a clear picture of receptor topology should help us to discover the structural determinants of its interaction with other proteins.

As sigma receptors bind antipsychotic drugs, understanding their mechanism of action might have practical implications. At the same time, channel regulation by protein-protein interactions deserves further attention, as it adds degrees of freedom to the way in which ion channels govern neuronal function.

Juan Carlos López

(2) References and links

ORIGINAL RESEARCH PAPER Aydar, E. et al. The sigma receptor as a ligand-regulated ORIGINAL RESEARCH PAPER Aydar, E. et al. The sigma recep
auxiliary potassium channel subunit. Neuron $\mathbf{3 4}, 399-410$ (2002)

FURTHER READING Lupardus, P. J. et al. Membrane-delimited coupling between sigma receptors and $\mathrm{K}^{+}$channels in rat neurohypophysial terminals requires neither $\mathrm{G}$-proteins nor ATP. J. Physiol. (Lond.) 526, 527-539 (2000)

WEB SITES

Encyclopedia of Life Sciences: http://www.els.net/

sodium, calcium and potassium channels | voltage-gated potassium channels 